\title{
Movimentação de metais pesados em Latossolo adubado com composto de lixo urbano(1)
}

\author{
Fernando Carvalho Oliveira(2), Maria Emilia Mattiazzo(3), Claudio Roberto Marciano(4) \\ e Cassio Hamilton Abreu Junior ${ }^{(5)}$
}

\begin{abstract}
Resumo - Em experimento realizado no campo, nos anos agrícolas 1996/97 e 1997/98, em Latossolo Amarelo distrófico, cultivado com cana-de-açúcar, avaliou-se o efeito de aplicações sucessivas de composto de lixo urbano sobre a movimentação, em profundidade, dos metais $\mathrm{Cd}, \mathrm{Cr}, \mathrm{Cu}, \mathrm{Ni}, \mathrm{Pb}$ e zinco. No primeiro ano agrícola, além do tratamento testemunha, o composto de lixo foi aplicado nas doses de 20, 40 e $60 \mathrm{Mg} \mathrm{ha}^{-1}$ (base seca). No segundo ano, o composto foi reaplicado nas doses de 24, 48 e $72 \mathrm{Mg} \mathrm{ha}^{-1}$. Em relação aos metais $\mathrm{Cd}, \mathrm{Cr}, \mathrm{Cu}, \mathrm{Ni}$ e $\mathrm{Pb}$ não se observou nenhuma evidência de movimentação ao longo do perfil do solo. O Zn apresentou mobilidade no solo, onde se verificou, ao final de 1997/98, incrementos significativos até a camada de 0,4-0,6 $\mathrm{m}$ de profundidade.
\end{abstract}

Termos para indexação: cobre, cromo, zinco.

\section{Movement of heavy metals in an Oxisol fertilized with municipal solid waste compost}

\begin{abstract}
From 1996 to 1998, a field experiment was carried out to study the effects of successive application of a municipal solid waste compost on the movement of $\mathrm{Cd}, \mathrm{Cr}, \mathrm{Cu}, \mathrm{Ni}, \mathrm{Pb}$, and $\mathrm{Zn}$ through the profile of a Yellow Latossol (Typic Hapludox) cropped with sugarcane. The compost was applied at rates of 20, 40 and $60 \mathrm{Mg} \mathrm{ha}^{-1}$ (dry base) in the first year, and of 24, 48 and $72 \mathrm{Mg} \mathrm{ha}^{-1}$ in the second year, respectively, plus a control treatment. The results demonstrated that there was no movement of $\mathrm{Cd}, \mathrm{Cr}, \mathrm{Cu}, \mathrm{Ni}$, and $\mathrm{Pb}$ through the profile of this acid soil. Nevertheless, $\mathrm{Zn}$ mobility was verified at the end of the second year, when content of $\mathrm{Zn}$ increased from 0.4 to $0.6 \mathrm{~m}$ layer.
\end{abstract}

Index terms: copper, chromium, zinc.

\section{Introdução}

A utilização do composto de lixo urbano na agricultura, como adubo orgânico e condicionador de solos, constitui importante alternativa para a gestão

(1) Aceito para publicação em 13 de agosto de 2002 .

Extraído da tese de doutorado do primeiro autor apresentada à Escola Superior de Agricultura Luiz de Queiroz (Esalq), Piracicaba, SP. Financiado pela Fapesp.

(2) Biossolo Agricultura \& Ambiente S.C. Ltda., Rua Edu Chaves, 822, São Dimas, CEP 13416-020 Piracicaba, SP. E-mail: fernando@biossolidos.com.br

(3) Esalq, Dep. de Ciências Exatas, Caixa Postal 9, CEP 13418-900 Piracicaba, SP. E-mail: mmatiazo@carpa.ciagri.usp.br

(4) Universidade Estadual do Norte Fluminense, Centro de Ciência e Tecnologia Agropecuária, Av. Alberto Lamego, 2000, Horto, CEP 28015-620 Campos dos Goytacazes, RJ. E-mail: crmarcia@bol.com.br

(5) Universidade de São Paulo, Centro de Energia Nuclear na Agricultura, Lab. de Nutrição Mineral de Plantas, Caixa Postal 96, CEP 13400-970 Piracicaba, SP. E-mail: cahabreu@cena.usp.br do lixo urbano, aliando reciclagem de nutrientes e destinação final adequada. No entanto, a maioria dos compostos de lixo apresenta em sua composição, além de nutrientes e matéria orgânica, organismos patogênicos, compostos orgânicos persistentes e tóxicos, e metais pesados potencialmente prejudiciais ao ambiente (Escosteguy et al., 1993; Grossi, 1993).

Os metais pesados podem expressar seu potencial poluente diretamente sobre os organismos dos solos, pela disponibilidade às plantas, pela contaminação de águas superficiais, via erosão do solo, e das águas subsuperficiais, por sua movimentação vertical e descendente no perfil do solo (Logan \& Chaney, 1983; Levine et al., 1989). Portanto, conhecer o destino desses elementos no solo é essencial para a avaliação do impacto ambiental provocado pelo uso agrícola de compostos de lixo urbano, uma vez que a extensão desse impacto está diretamente relacionada com a habilidade do solo em reter esses metais (Elliott et al., 1986). 
Sawhney et al. (1994) estudaram o potencial de lixiviação de metais pesados pela utilização, em substratos no cultivo de plantas ornamentais, de composto de lixo em doses equivalentes a $0 \%, 25 \%$, $50 \%$ e $100 \%$ em volume, utilizando uma mistura $(\mathrm{pH}$ 6,5) de turfa, areia e calcário como material de enchimento. As concentrações de $\mathrm{Cd}, \mathrm{Cr}, \mathrm{Cu}, \mathrm{Ni}, \mathrm{Pb}$ e $\mathrm{Zn}$ nos lixiviados aumentaram com a proporção utilizada do resíduo, mas decresceram no decorrer das lixiviações. Os maiores teores foram encontrados nas amostras coletadas nas duas primeiras semanas, os quais foram atribuídos às formas solúveis ou trocáveis de metais presentes no composto de lixo. Foram verificados, respectivamente, entre as doses de $0 \%$ e $100 \%$, as seguintes variações: 0,25 a

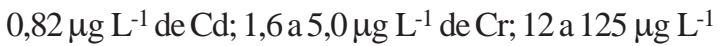
de $\mathrm{Cu} ; 13$ a $31 \mu \mathrm{g} \mathrm{L}{ }^{-1}$ de Ni; 1,9 a $17 \mu \mathrm{g} \mathrm{L}^{-1}$ de Pb; e 54 a $219 \mu \mathrm{g} \mathrm{L}^{-1}$ de zinco. Esses valores estiveram abaixo dos estabelecidos para água potável pela United States Environmental Protection Agency (40 CFR Part 503) (Estados Unidos, 1993), ou seja, $\leq 5 \mu \mathrm{g} \mathrm{L}^{-1}$ de $\mathrm{Cd} ; \leq 50 \mu \mathrm{g} \mathrm{L}^{-1}$ de $\mathrm{Cr} ; \leq 1.300 \mu \mathrm{g} \mathrm{L}^{-1}$ de $\mathrm{Cu}$; $\leq 100 \mu \mathrm{g} \mathrm{L}^{-1} \mathrm{de} \mathrm{Ni} ; \leq 20 \mu \mathrm{g} \mathrm{L}{ }^{-1}$ de chumbo. Com relação ao $\mathrm{Zn}$, não são estabelecidos valores críticos. Os autores concluíram que a lixiviação de metais pesados para águas de subsuperfície, em solos agrícolas tratados com composto de lixo, pode ser considerada desprezível, porque as ínfimas quantidades de metais lixiviadas do composto deverão ter sua mobilidade atenuada pelas camadas do subsolo.

Em solos de regiões tropicais existem muitas dúvidas a respeito da mobilidade dos metais pesados, justificadas, em parte, pela carência de estudos de longo prazo, agravados pela tendência de grande mobilidade desses metais, já que nessas regiões pre- dominam solos altamente intemperizados sob regimes de temperatura e precipitações pluviais elevadas.

O objetivo deste trabalho foi estudar a movimentação de metais pesados em profundidade, num Latossolo Amarelo distrófico tratado com aplicações sucessivas de composto de lixo urbano.

\section{Material e Métodos}

O experimento foi realizado nos anos agrícolas 1996/97 e 1997/98, numa área plana de Latossolo Amarelo distrófico da Estação Experimental do Instituto Agronômico de Campinas, em Piracicaba, SP. O clima, de acordo com a classificação de Köppen, é do tipo Cwa: subtropical com inverno seco e com as seguintes médias anuais: precipitação, $1.253 \mathrm{~mm}$; temperatura, $21,1^{\circ} \mathrm{C}$; umidade relativa do ar, $74 \%$; velocidade do vento, $2,2 \mathrm{~m} \mathrm{~s}^{-1}$. Algumas características químicas e físicas do solo são apresentadas na Tabela 1.

Foram empregadas duas remessas de composto de lixo urbano, originário da usina de tratamento de lixo da cidade de São Jorge, Prefeitura Municipal de Santo André, SP. A caracterização química parcial dos resíduos (Tabela 2) foi realizada de acordo com Eaton et al. (1995).

Estabeleceram-se quatro tratamentos num delineamento em blocos casualizados, com quatro repetições, em parcelas experimentais de $100 \mathrm{~m}^{2}$ cultivadas com cana-de-açúcar em ciclo anual. Os tratamentos foram: testemunha absoluta (T), composto de lixo nas doses A(CLa), B (CLb) e C (CLc). As doses de composto de lixo (base seca) foram, na primeira aplicação (1996/97), de 20 (A), 40 (B) e $60 \mathrm{Mg} \mathrm{ha}^{-1}$ (C), e na segunda (1997/98), de 24 (A), 48 (B) e $72 \mathrm{Mg} \mathrm{ha}^{-1}$ (C). A primeira aplicação foi feita na superfície do solo em área total, com distribuição manual nas parcelas e incorporação, na camada de 0-0,2 m, com enxada rotativa. Na segunda aplicação, a incorporação foi

Tabela 1. Características químicas e físicas do Latossolo Amarelo distrófico antes da instalação do experimento.

\begin{tabular}{|c|c|c|c|c|c|c|c|c|c|c|c|}
\hline $\begin{array}{l}\text { Profundidade } \\
\text { (m) }\end{array}$ & $\begin{array}{c}\mathrm{pH} \\
\left(\mathrm{CaCl}_{2}\right)\end{array}$ & $\begin{array}{c}\text { C-org } \\
\left(\mathrm{g} \mathrm{kg}^{-1}\right)\end{array}$ & $\begin{array}{c}\mathrm{P} \\
\left(\mathrm{mg} \mathrm{dm}^{-3}\right)\end{array}$ & $\mathrm{K}$ & $\mathrm{Ca}$ & $\mathrm{Mg}$ & $\begin{array}{l}\mathrm{H}+\mathrm{Al} \\
\mathrm{ol}_{\mathrm{c}} \mathrm{dm}\end{array}$ & $\mathrm{Al}$ & SB & $\begin{array}{l}\mathrm{T} \\
---\end{array}$ & $\begin{array}{c}\mathrm{V} \\
(\%)\end{array}$ \\
\hline $0,0-0,2$ & 4,1 & 9,3 & 3 & 0,3 & 11 & 5 & 47 & 10 & 16,3 & 63,3 & 25,7 \\
\hline $0,2-0,4$ & 4,1 & 8,1 & 2 & 0,3 & 14 & 5 & 47 & 10 & 19,3 & 66,3 & 29,1 \\
\hline $0,4-0,6$ & 4,1 & 6,4 & 1 & 0,1 & 11 & 3 & 47 & 12 & 14,1 & 61,1 & 23,1 \\
\hline & $\mathrm{Al}_{2} \mathrm{O}_{3}$ & $\begin{array}{r}\mathrm{Fe}_{2} \mathrm{O}_{3} \\
-\left(\mathrm{g} \mathrm{kg}^{-1}\right) \\
\end{array}$ & $\mathrm{SiO}_{2}$ & \multicolumn{2}{|c|}{ Areia grossa } & Areia fina & \multicolumn{2}{|c|}{ Silte } & Argila & \multicolumn{2}{|c|}{$\begin{array}{c}\text { Densidade } \\
\left(\mathrm{kg} \mathrm{m}^{-3}\right)\end{array}$} \\
\hline $0,0-0,2$ & 108 & 71 & 121 & \multicolumn{2}{|c|}{195} & 329 & \multicolumn{2}{|c|}{141} & 335 & \multicolumn{2}{|c|}{1.316} \\
\hline $0,2-0,4$ & 144 & 86 & 148 & \multicolumn{2}{|c|}{194} & 248 & \multicolumn{2}{|c|}{116} & 442 & \multicolumn{2}{|c|}{1.292} \\
\hline $0,4-0,6$ & 150 & 89 & 155 & \multicolumn{2}{|c|}{211} & 218 & \multicolumn{2}{|c|}{90} & 481 & \multicolumn{2}{|c|}{1.277} \\
\hline
\end{tabular}

(1)Densidade de partículas. 
realizada nas entrelinhas da cultura. O plantio da cana-de-açúcar, variedade RB-78-5148, foi realizado após 50 dias da incorporação do composto de lixo urbano.

A movimentação de $\mathrm{Cd}, \mathrm{Cr}, \mathrm{Cu}, \mathrm{Ni}, \mathrm{Pb}$ e $\mathrm{Zn}$ no perfil do solo foi avaliada por meio de dois procedimentos. No primeiro, a determinação desses elementos foi realizada em amostras de solução do solo coletadas, periodicamente, durante o ano agrícola 1997/98. Para tanto, após a incorporação dos resíduos (segunda aplicação), foram instaladas 16 baterias de três unidades extratoras de solução do solo, uma por parcela experimental, a 0,40 $\mathrm{m}$ da linha central, nas profundidades de 0,3, 0,6 e 0,9 m. As cápsulas porosas foram previamente lavadas em solução $0,1 \mathrm{~mol} \mathrm{~L}^{-1}$ de $\mathrm{HCl}$ e, em seguida, as unidades extratoras foram testadas quanto a possíveis vazamentos mediante imersão das peças em água destilada. A unidade extratora de solução do solo foi a mesma descrita por Reichardt et al. (1977). As extrações da solução do solo foram realizadas em razão da ocorrência das chuvas a partir da incorporação da segunda aplicação do composto de lixo urbano (13/11/1997). O volume extraído variou com a umidade do solo e, por esta razão, as amostras analisadas foram formadas por uma, duas ou três extrações em sequiência. Assim, num período de 408 dias de monitoramento foram consideradas a composição das extrações realizadas de 33 a 45, 91 a 96, 174 a 175, 208 a 209, 312 a 313, 341 a 342 e 395 a 396 dias após a incorporação do composto de lixo urbano.

O segundo procedimento foi a determinação dos teores totais dos metais pesados em amostras de solo coletadas dentro da área útil das parcelas $\left(25,2 \mathrm{~m}^{2}\right.$ centrais $)$, nas camadas 0-0,2, 0,2-0,4, 0,4-0,6 e 0,6-0,8 m, aos 360 dias após a incorporação de cada aplicação de composto de lixo. Cada amostra era composta de cinco subamostras coletadas nas entrelinhas da cultura. Os metais foram extraídos em água-régia com digestão em forno de microon-

Tabela 2. Composição química parcial das duas remessas de composto de lixo aplicadas no solo em 1996 e $1997^{(1)}$.

\begin{tabular}{lrr}
\hline Atributo analisado & 1996 & 1997 \\
\hline $\mathrm{pH}\left(\mathrm{H}_{2} \mathrm{O}, 1: 2,5\right)$ & 4,8 & 8,0 \\
Carbono orgânico $\left(\mathrm{g} \mathrm{kg}^{-1}\right)$ & 342 & 282 \\
$\mathrm{~N}$ total $\left(\mathrm{g} \mathrm{kg}^{-1}\right)$ & 10 & 13 \\
Relação C/N-total & 34 & 21 \\
$\mathrm{Cd}$ total $\left(\mathrm{mg} \mathrm{kg}^{-1}\right)$ & $\mathrm{nd}$ & $\mathrm{nd}$ \\
$\mathrm{Cr}$ total $\left(\mathrm{mg} \mathrm{kg}^{-1}\right)$ & 41 & 81 \\
$\mathrm{Cu}$ total $\left(\mathrm{mg} \mathrm{kg}^{-1}\right)$ & 223 & 403 \\
$\mathrm{Ni}$ total $\left(\mathrm{mg} \mathrm{kg}^{-1}\right)$ & 26 & 34 \\
$\mathrm{~Pb}$ total $\left(\mathrm{mg} \mathrm{kg}^{-1}\right)$ & 92 & 196 \\
Zn total $\left(\mathrm{mg} \mathrm{kg}^{-1}\right)$ & 387 & 496 \\
\hline
\end{tabular}

(1)Exceto o pH, cuja determinação foi feita a partir do material úmido, os demais resultados são expressos com base no material seco; nd: valores abaixo do limite de determinação do método analítico empregado ( $<9 \mathrm{mg} \mathrm{kg}^{-1}$ na amostra); os dados representam as médias de três amostras compostas coletadas de cada remessa do composto de lixo urbano. das (CEM - modelo MDS-2000), conforme Nieuwenhuize et al. (1991). Foram utilizados $500 \mathrm{mg}$ de terra, sendo o extrato recuperado com água bidestilada em $50 \mathrm{~mL}$ de volume final.

As determinações analíticas dos metais pesados na solução do solo e nos extratos de solo foram feitas por espectrometria de absorção atômica com chama convencional ar/acetileno.

Os dados obtidos foram analisados em delineamento com parcelas subdivididas. Os tratamentos (T, CLa, CLb e CLc) foram considerados como parcelas e as profundidades de coleta das amostras, as subparcelas. Quando constatada interação significativa pelo teste $\mathrm{F}$, as médias foram testadas, dentro de profundidades, por modelos de regressão polinomial de $1^{\mathrm{o}}$ e $2^{\mathrm{o}}$ graus e, dentro de tratamentos, pelo teste de Tukey a $5 \%$ de probabilidade.

\section{Resultados e Discussão}

Os teores dos metais $\mathrm{Cd}^{2+}, \mathrm{Cr}, \mathrm{Cu}^{2+}, \mathrm{Ni}^{2+}, \mathrm{Pb}^{2+} \mathrm{e}$ $\mathrm{Zn}^{2+}$ avaliados em todas as amostras de solução do solo, coletadas nas profundidades de 0,3, 0,6 e 0,9 m, estiveram abaixo dos limites de determinação analítica do método empregado, ou seja, os teores de $\mathrm{Cd}^{2+}$ foram $<0,08 \mathrm{mg} \mathrm{L}^{-1}$; de Cre $\mathrm{Ci}^{2+},<0,10 \mathrm{mg} \mathrm{L}^{-1} ; \mathrm{de} \mathrm{Cu}^{2+}$

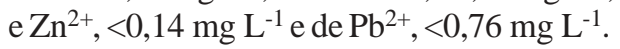

Sawhney et al. (1994) demonstraram que as concentrações de metais pesados em águas lixiviadas, por substratos à base de composto de lixo, são extremamente baixas, o que dificulta sua determinação analítica.

Todavia algumas considerações sobre o método de obtenção das amostras de solução do solo devem ser salientadas. Segundo Suarez (1987), a extração por meio de vácuo pode levar a erros, uma vez que nesse processo ocorre a eliminação de $\mathrm{CO}_{2} \mathrm{e}$ outros compostos voláteis, propiciando aumentos no pH da amostra. De fato, não se sabe se apenas por esta razão, os valores de $\mathrm{pH}$ das amostras da solução do solo coletadas na $1 \underline{a}$ e $7 \underline{a}$ amostragens apresentaram, respectivamente, médias de 7,6 $6 \pm 0,1 \mathrm{e}$ $8,2 \pm 0,02$, independentemente dos tratamentos e profundidades de coleta estabelecidos. Moraes \& Dynia (1990) verificaram durante a coleta da solução do solo, através de extratores, progressiva obstrução dos poros das cápsulas com partículas coloidais do solo e a conseqüente diminuição na taxa de infiltração da solução. Também observaram a formação de uma capa coloidal de alta reatividade na superfície 
das cápsulas que, por causa da elevação do $\mathrm{pH}$ da amostra, tornaria possível a precipitação de óxidos/ hidróxidos de ferro na sua superfície interna, ocasionando um ambiente propício para ocorrência de processos de adsorção e co-precipitação, podendo, assim, modificar a concentração de outros cátions metálicos. Tais observações também foram apresentadas por Oliveira \& Mattiazzo (2001) para explicar resultados semelhantes aos do presente trabalho, obtidos no mesmo tipo de solo onde foram adicionadas doses crescentes de lodo de esgoto.

Nas amostras de solo coletadas em profundidade não foram detectados os metais $\mathrm{Cd}$, $\mathrm{Ni}$ e $\mathrm{Pb}$, por estarem abaixo dos limites de determinação analítica, ou seja, suas concentrações no solo foram, respectivamente, menores que 8,10 e $76 \mathrm{mg} \mathrm{kg}^{-1}$. Porém, é preciso considerar que as concentrações desses metais no solo, proporcionadas pela aplicação do resíduo até mesmo nas maiores doses, são baixas (1,5 e 7,5 mg kg-1 em relação ao $\mathrm{Ni}$ e $\mathrm{Pb}$, respectivamente) e, portanto, de difícil avaliação num experimento realizado no campo.

Quanto aos metais $\mathrm{Cu}$ e $\mathrm{Cr}$, a aplicação de doses crescentes do composto de lixo não proporcionou incrementos nos teores das camadas de solo abaixo de 0-0,2 m, em ambos os anos agrícolas (Tabela 3). De maneira geral, dentro de cada tratamento em profundidade, os teores médios de $\mathrm{Cu}$ e $\mathrm{Cr}$ ocorreram naturalmente nas camadas de 0,4-0,6 e 0,6-0,8 m na maioria dos casos, em concentrações iguais ou maiores do que nas camadas onde foram incorporadas as doses do resíduo. Por causa disso, é possível afirmar que não houve evidências da mobilidade do $\mathrm{Cu}$ e do $\mathrm{Cr}$ ao final dos dois anos experimentais.

Tabela 3. Teores totais de $\mathrm{Cu}, \mathrm{Cr}$ e $\mathrm{Zn}$ determinados no solo, nas várias profundidades, em razão da aplicação de doses de composto de lixo urbano nos anos agrícolas 1996/97 e 1997/98(1).

\begin{tabular}{|c|c|c|c|c|c|c|}
\hline \multirow{2}{*}{$\begin{array}{l}\text { Profundidade } \\
\text { (m) }\end{array}$} & \multicolumn{4}{|c|}{ Composto de lixo $\left(\mathrm{Mg} \mathrm{ha}^{-1}\right)^{(2)}$} & \multicolumn{2}{|c|}{ Coeficiente de determinação } \\
\hline & 0 & $20(24)$ & $40(48)$ & $60(72)$ & Linear & Quadrático \\
\hline & \multicolumn{6}{|c|}{$\mathrm{Cu}\left(\mathrm{mg} \mathrm{kg}^{-1}\right)-$ Ano agrícola $1996 / 97$} \\
\hline $0,0-0,2$ & $17,9 b$ & $20,1 b$ & $22,4 a b$ & $22,3 a$ & $0,88^{*}$ & ns \\
\hline $0,2-0,4$ & $19,1 b$ & $19,5 b$ & $20,5 b$ & $19,4 b$ & ns & ns \\
\hline $0,4-0,6$ & $21,8 \mathrm{a}$ & $22,7 \mathrm{a}$ & $23,6 \mathrm{a}$ & $23,6 \mathrm{a}$ & ns & ns \\
\hline \multirow[t]{2}{*}{$0,6-0,8$} & $21,5 \mathrm{a}$ & $21,4 \mathrm{ab}$ & $22,2 \mathrm{ab}$ & $22,1 \mathrm{a}$ & ns & ns \\
\hline & \multicolumn{6}{|c|}{$\mathrm{Cu}\left(\mathrm{mg} \mathrm{kg}^{-1}\right)-$ Ano agrícola $1997 / 98$} \\
\hline $0,0-0,2$ & $16,9 \mathrm{~b}$ & $21,4 \mathrm{a}$ & $26,7 \mathrm{a}$ & $31,7 \mathrm{a}$ & $0,99^{* *}$ & ns \\
\hline $0,2-0,4$ & $19,1 b$ & $19,8 \mathrm{a}$ & $18,6 \mathrm{c}$ & $19,1 b$ & ns & ns \\
\hline $0,4-0,6$ & $22,7 \mathrm{a}$ & $22,9 \mathrm{a}$ & $24,7 \mathrm{ab}$ & $22,6 b$ & ns & ns \\
\hline \multirow[t]{2}{*}{$0,6-0,8$} & $22,9 \mathrm{a}$ & $22,3 \mathrm{a}$ & $22,7 \mathrm{~b}$ & $21,3 b$ & ns & ns \\
\hline & \multicolumn{6}{|c|}{$\mathrm{Cr}\left(\mathrm{mg} \mathrm{kg}^{-1}\right)-$ Ano agrícola 1996/97 } \\
\hline $0,0-0,2$ & 16,6 & 17,7 & 17,5 & 17,6 & ns & ns \\
\hline $0,2-0,4$ & 20,0 & 22,6 & 21,7 & 19,7 & ns & ns \\
\hline $0,4-0,6$ & 22,6 & 22,7 & 23,2 & 23,0 & ns & ns \\
\hline \multirow[t]{2}{*}{$0,6-0,8$} & 24,2 & 25,4 & 25,7 & 25,3 & ns & ns \\
\hline & \multicolumn{6}{|c|}{$\mathrm{Cr}\left(\mathrm{mg} \mathrm{kg}^{-1}\right)-$ Ano agrícola $1997 / 98$} \\
\hline $0,0-0,2$ & $15,6 b$ & $17,5 b$ & $19,5 b$ & $19,5 b$ & $0,89^{*}$ & ns \\
\hline $0,2-0,4$ & $18,8 \mathrm{~b}$ & $22,4 b$ & $19,9 \mathrm{~b}$ & $18,4 \mathrm{~b}$ & $\mathrm{~ns}$ & ns \\
\hline $0,4-0,6$ & $23,1 \mathrm{a}$ & $22,9 \mathrm{a}$ & $26,0 \mathrm{a}$ & $25,0 \mathrm{a}$ & ns & ns \\
\hline \multirow[t]{2}{*}{$0,6-0,8$} & $25,6 \mathrm{a}$ & $25,1 \mathrm{a}$ & $25,8 \mathrm{a}$ & $24,6 \mathrm{a}$ & ns & ns \\
\hline & \multicolumn{6}{|c|}{$\mathrm{Zn}\left(\mathrm{mg} \mathrm{kg}^{-1}\right)-$ Ano agrícola $1996 / 97$} \\
\hline $0,0-0,2$ & $21,1 b$ & $23,6 a$ & $31,4 \mathrm{a}$ & $36,3 \mathrm{a}$ & $0,97^{* *}$ & ns \\
\hline $0,2-0,4$ & $19,7 \mathrm{~b}$ & $19,5 b$ & $25,5 b$ & $25,1 \mathrm{~b}$ & $0,75^{* *}$ & ns \\
\hline $0,4-0,6$ & $22,8 \mathrm{ab}$ & $23,5 \mathrm{a}$ & $26,0 \mathrm{~b}$ & $24,5 \mathrm{~b}$ & ns & ns \\
\hline \multirow[t]{2}{*}{$0,6-0,8$} & $25,5 \mathrm{a}$ & $26,7 \mathrm{a}$ & $27,8 \mathrm{ab}$ & $27,3 b$ & ns & ns \\
\hline & \multicolumn{6}{|c|}{$\mathrm{Zn}\left(\mathrm{mg} \mathrm{kg}^{-1}\right)-$ Ano agrícola $1997 / 98$} \\
\hline $0,0-0,2$ & $20,2 b$ & $34,8 \mathrm{a}$ & $45,9 \mathrm{a}$ & $51,6 \mathrm{a}$ & $0,97^{* *}$ & $0,99^{* *}$ \\
\hline $0,2-0,4$ & $19,6 b$ & $26,5 b$ & $28,0 \mathrm{~b}$ & $27,4 b$ & $0,68^{\text {** }}$ & $0,99^{* *}$ \\
\hline $0,4-0,6$ & $22,1 \mathrm{ab}$ & $29,2 b$ & $29,4 b$ & $28,7 \mathrm{~b}$ & $0,54^{* *}$ & $0,95^{* *}$ \\
\hline $0,6-0,8$ & $26,2 \mathrm{a}$ & $27,8 \mathrm{~b}$ & $27,7 \mathrm{~b}$ & $26,7 b$ & ns & ns \\
\hline
\end{tabular}

${ }^{(1)}$ Para cada metal, dentro de cada ano agrícola, médias seguidas da mesma letra, em cada coluna, não diferem entre si pelo teste de Tukey a $5 \%$ de probabilidade. ${ }^{(2)}$ Doses entre parênteses referem-se ao ano agrícola $1997 / 98$. ns Não-significativo. * e **Significativo a $5 \%$ e a $1 \%$, respectivamente, pelo teste F. 
A imobilidade do Cu também foi observada por Williams et al. (1987), Dowdy et al. (1991), Chino et al. (1992) e Oliveira \& Mattiazzo (2001) em solos tratados com elevadas doses de lodo de esgoto e monitorados por períodos de, respectivamente, 9,14 , 12 e 2 anos. A imobilidade do Cu normalmente é atribuída à formação de complexos organometálicos estáveis de baixa solubilidade (Taylor et al., 1995) e à ligação do metal às frações não trocáveis do solo, a chamada fração residual e a óxidos de Fe e Mn (Matos et al., 1996; Amaral Sobrinho et al., 1997). Outro fator a ser considerado é o aumento do $\mathrm{pH}$ do solo, em conseqüência da aplicação do composto de lixo (Abreu Junior et al., 2000; Oliveira et al., 2002), que favorece reações de precipitação e complexação dos metais, reduzindo sua solubilidade e mobilidade.

Com relação ao Cr, os resultados foram semelhantes aos de Williams et al. (1987), Bertoncini (1997) e Oliveira \& Mattiazzo (2001) em solos tratados com lodo de esgoto. Esses autores atribuíram a imobilidade do $\mathrm{Cr}$ à sua presença na forma trivalente $\left(\mathrm{Cr}^{+3}\right)$, a qual poderia ter sido precipitada como hidróxidos de fórmulas genéricas $\mathrm{Cr}(\mathrm{OH})_{3}$ ou $\mathrm{Fe}_{\mathrm{x}} \mathrm{Cr}_{1-\mathrm{x}}(\mathrm{OH})_{3} \mathrm{em}$ meio alcalino ou pouco ácido, complexado com moléculas orgânicas pouco solúveis ou adsorvido à superfície dos minerais.

Com relação ao Zn, no ano agrícola 1996/97, os teores totais no solo aumentaram linearmente, em razão das doses de composto de lixo, até a camada de 0,2-0,4 m (Tabela 3). Em 1997/98, esses aumentos foram observados até a camada de 0,4-0,6 m, evidenciando uma progressão dos teores em profundidade, com as aplicações sucessivas do composto de lixo.

Comparando-se os teores de $\mathrm{Zn}$ entre camadas do solo, dentro dos tratamentos CLa, CLb e CLc, os maiores valores foram obtidos na camada de incorporação, e abaixo de $0,2 \mathrm{~m}$, os teores foram praticamente iguais. Dessa forma, embora tenham sido observados aumentos nos teores naturais de $\mathrm{Zn}$ do solo em razão da profundidade de coleta das amostras, no ano agrícola 1997/98, os acréscimos nos teores de $\mathrm{Zn}$ das camadas de 0,2-0,4 e 0,4-0,6 m, por causa da aplicação de composto de lixo, ficaram, respectivamente, em torno de $39 \%$ e $32 \%$ quando comparados com a testemunha, o que mostra que de fato ocorreu movimentação desse elemento no perfil do solo.
A movimentação do $\mathrm{Zn}$ em profundidade também foi observada em solos tratados com lodo de esgoto (Welch \& Lund, 1989; Oliveira \& Mattiazzo, 2001) e resíduos ácidos de siderurgia (Amaral Sobrinho et al., 1998). Segundo Kabata-Pendias \& Adriano (1995) e Amaral Sobrinho et al. (1998), a mobilidade do Zn está relacionada à sua presença no solo, predominantemente, em formas trocáveis e, portanto, facilmente solúveis.

Além de o Zn ter sido o metal adicionado ao solo em maior quantidade, sua lixiviação pode ter sido favorecida pela acidez do solo ( $\mathrm{pH} 4,5$ a 5,0), essencialmente no primeiro ano agrícola (Oliveira et al., 2002), o que estimularia a sua ocorrência em formas trocáveis e solúveis.

\section{Conclusões}

1. Após duas aplicações em anos sucessivos de composto de lixo urbano, não há evidências de movimentação, no perfil do solo, dos metais cobre e crômio.

2. O zinco se apresenta como um elemento móvel no perfil do solo, com grande potencial para atingir as águas subterrâneas por ocasião de aplicações sucessivas de composto de lixo urbano.

\section{Referências}

ABREU JUNIOR, C. H.; MURAOKA, T.; LAVORANTE, A. F.; ALVAREZ VENEGAS, F. C. Condutividade elétrica, reação do solo e acidez potencial em solos adubados com composto de lixo. Revista Brasileira de Ciência do Solo, Viçosa, MG, v. 24, n. 3, p. 635$647,2000$.

AMARAL SOBRINHO, N. M. B.; VELLOSO, A. C. X.; COSTA, L. M.; OLIVEIRA, C. Mobilidade de metais pesados em solo tratado com resíduo siderúrgico. Revista Brasileira de Ciência do Solo, Viçosa, MG, v. 22, n. 2, p. 345-353, 1998.

AMARAL SOBRINHO, N. M. B.; VELLOSO, A. C. X.; OLIVEIRA, C. Solubilidade de metais pesados em solo tratado com resíduo siderúrgico. Revista Brasileira de Ciência do Solo, Viçosa, MG, v. 21, n. 1, p. 9-16, 1997. 
BERTONCINI, E. I. Mobilidade de metais pesados em solos tratados com lodo de esgoto. 1997. 90 f. Dissertação (Mestrado em Solos e Nutrição de Plantas) - Escola Superior de Agricultura Luiz de Queiroz, Piracicaba, 1997.

CHINO, M.; SHIGEKO, G.; KUMAZAWA, K.; OWA, N.; YOSHIOKA, O.; TAKECHI, N.; INANAGA, S.; INOU, H.; DE-LONG, C.; YOUSSEF, R. A. Behavior of zinc and copper in soil with long term application of sewage sludges. Soil Science and Plant Nutrition, Tokyo, v. 38, n. 1, p. 159-167, 1992.

DOWDY, R. H.; LATTERELL, J. J.; HINESLY, T. D.; GROSSMAN, R. B.; SULLIVAN, D. L. Trace metal movement in an aeric Ochraqualf following 14 years of annual sludge applications. Journal of Environmental Quality, Madison, v. 20, n. 1, p. 119-123, 1991.

EATON, A. D.; CLESCERI, L. S.; GRENNBERG, A. E. Standard methods for the examination of water and wastewater. 19th ed. Washington: American Public Health Association/American Water Works Association/Water Environment Federation, 1995. 1082 p.

ELLIOTT, H. A.; LIBERATI, M. R.; HUANG, C. P. Competitive adsorption of heavy metals by soils. Journal of Environmental Quality, Madison, v. 15, n. 3, p. 214217, 1986.

Escosteguy, P. A. V.; PARChen, C. A. P.; SELBACH, P. A. Bactérias enteropatogênicas em compostos de lixo domiciliar, solo e planta. Revista Brasileira de Ciência do Solo, Campinas, v. 17, p. 365-369, 1993.

ESTADOS UNIDOS. Environmental Protection Agency. Title 40 CFR-Part 503 final rules: standards for the use or disposal of sewage sludge. Washington, 1993. p. 93879415. (Federal Register, 58).

GROSSI, M. G. de L. Avaliação da qualidade dos produtos obtidos de usinas de compostagem brasileiras de lixo doméstico através da determinação de metais pesados e substâncias orgânicas tóxicas. 1993. 222 f. Tese (Doutorado em Química Analítica) - Instituto de Química, Universidade de São Paulo, São Paulo, 1993.

KABATA-PENDIAS, A.; ADRIANO, D. C. Trace metals. In: RECHCIGL, J. E. (Ed.). Soil amendments and environmental quality. Boca Raton: Lewis, 1995. p. 139167.

LEVINE, M. B.; HALL, A. T.; BARRETT, G. W.; TAYLOR, D. H. Heavy metal concentrations during ten years of sludge treatment to an old-field community. Journal of Environmental Quality, Madison, v. 18, n. 4, p. 411-418, 1989.

LOGAN, T. J.; CHANEY, R. L. Metals. In: WORKSHOP ON UTILIZATION OF MUNICIPAL WASTEWATER AND SLUDGE ON LAND, 1983, Riverside. Proceedings... Riverside: University of California, 1983. p. 235-323.

MATOS, A. T.; FONTES, M. P. F.; JORDÃO, C. P.; COSTA, L. M. Mobilidade e formas de retenção de metais pesados em Latossolo Vermelho Amarelo. Revista Brasileira de Ciência do Solo, Viçosa, MG, v. 20, p. 379-386, 1996.

MORAES, J. F. V.; DYNIA, J. F. Uso de cápsulas porosas para extrair solução do solo. Pesquisa Agropecuária Brasileira, Brasília, v. 25, n. 10, p. 1523-1528, out. 1990.

NIEUWENHUIZE, J.; POLEY-VOS, C. H.; AKKER, A. H. van den; DELFT, W. van. Comparison of microwave and conventional extraction techniques for the determination of metals in soil sediment and sludge samples by atomic spectrometry. Analyst, London, v. 116, p. 347$351,1991$.

OLIVEIRA, F. C.; MATTIAZZO, M. E. Mobilidade de metais pesados em um Latossolo Amarelo distrófico tratado com lodo de esgoto e cultivado com cana-de-açúcar. Scientia Agricola, Piracicaba, v. 58, n. 4, p. 807-812, 2001.

OLIVEIRA, F. C.; MATTIAZZO, M. E.; MARCIANO, C. R.; ABREU JUNIOR, C. H. Alterações em atributos químicos de um Latossolo pela aplicação de composto de lixo urbano. Pesquisa Agropecuária Brasileira, Brasília, v. 37, n. 4, p. 529-538, abr. 2002.

REICHARDT, K.; LIBARDI, P. L.; MEIRELLES, N. M. F.; FERREYRA, F. F. H.; ZAGATTO, E. A. G.; MATSUI, E. Extração e análise de nitratos em solução do solo. Revista Brasileira de Ciência do Solo, Campinas, v. 1, p. 130-132, 1977. 
SAWHNEY, B. L.; BUGBEE, G. T.; STILWELL, D. E. Leachability of heavy metals from growth media containing source-separated municipal solid waste compost. Journal of Environmental Quality, Madison, v. 23, p. 718-722, 1994.

SUAREZ, D. L. Prediction of $\mathrm{pH}$ errors in soil: water extractors due to degassing. Soil Science Society of America Journal, Madison, v. 51, p. 64-67, 1987.

TAYLOR, R. W.; XIU, H.; MEHADI, A. A.; SHUFORD, J. W.; TADESSE, W. Fractionation of residual cadmium, copper, nickel, lead, and zinc in previously sludge-amended soil. Communications in Soil Science and Plant Analysis, New York, v. 26, n. 13/14, p. 2193-2204, 1995.

WELCH, J. E.; LUND, L. J. Zinc movement in sewagesludge-treated soil as influenced by soil properties, irrigation water quality, and soil moisture level. Soil Science, New Brunswick, v. 147, n. 3, p. 208-214, 1989.

WILLIAMS, D. E.; VLAMIS, J.; PUKITE, A. H.; COREY, J. E. Metal movement in sludge-amended soils: a nine-year study. Soil Science, New Brunswick, v. 143, n. 2, p. 124-131, 1987. 